\title{
MEDIDA DA DIFERENÇA ARTÉRIO-VENOSA DE OXIGÊNIO NA MONITORIZAÇÃO DE PACIENTES COM HEMORRAGIA SUBARACNÓIDEA POR ANEURISMA CEREBRAL
}

\author{
RONALDO SÉRGIO SANTANA PEREIRA * MÁRCIO ROBERTTI RAMALHO***, \\ RENATO CAMARGO VISCARDI**, LUIZ AUGUSTO CASULARI ROXO DA MOTTA*, \\ MÁRCIO VINHAL DE CARVALHO***, KUNIO SUZUKI*, PAULO ANDRADE DE MEULO***
}

\begin{abstract}
RESUMO - A diferença artério-venosa de oxigênio (DAVO2), pelo fato de estar relacionada com o metabolismo cerebral, reflete alteraçōes que ocorrem em determinadas situaçōes patológicas, entre elas as causadas pela hemorragia subaracnoidea espontânea (HSAE). Com a finalidade de avaliar a relação entre alteraçð̄es na DAVO2 com o quadro clínico e a evolução de pacientes com HSAE, devido à ruptura de aneurisma cerebral, este método foi utilizado em 30 pacientes portadores desta patologia, admitidos na Unidade de Neurocirurgia do HBDF. A HSAE foi confirmada por CT de crânio em 17 pacientes e por punção lombar em 13. Dezoito pacientes foram admitidos com Hunt \& Hess (H\&H) I ou II, sete com H\&H III e cinco com H\&H IV ou V. A medida da DAVO2 baseou-se na equação de Fick e os resultados clínicos foram avaliados pela escala de sequelas de Glasgow. Dezenove pacientes apresentaram DAVO2 normais (inicialmente e durante a evoluçåo), sendo que três faleceram; cinco tiveram valores de DAVO2 sempre baixos e três faleceram; os restantes seis pacientes tiveram valores da DAVO2 sempre elevados e dois faleceram. Os pacientes com DAVO2 normais tiveram melhor evoluçāo clínica e índice de mortalidade menor, quando comparados com os pacientes com valores anomais da DAVO2 ( $p<0,05$ ). O diagnóstico de vasoespasmo foi feito, em sua maioria, pela avaliação clínica, sendo confirmado radiologicamente em oito pacientes, mas estes últimos tiveram DAVO2 normais. A medida isolada da DAVO2 não foi suficiente para o diagnóstico do vasoespasmo, porem refletiu alterações metabólicas precoces. Baseada nestes resultados, a DAVO2 pode ser usada como parâmetro importante na monitorizaçāo da evoluçāo dos pacientes com HSAE.
\end{abstract}

PALAVRAS-CHAVE: hemorragia subaracnóidea espontânea, aneurisma cerebral, vasoespasmo cerebral, fluxo sanguíneo cerebral, diferença artério-venosa de oxigênio (DAVO2).

Measurement of arteriovenous oxygen difference in the monitoring of patients with subarachnoid haemorrhage due to cerebral aneurysm

ABSTRACT - The arterious venous oxygen difference (AVDO2) due to the close relationship with cerebral metabolic rate of oxygen and cerebral blood flow shows metabolic alterations that occur in some pathological situations in the brain including subarachnoid haemorrhage. The AVDO2 was calculated by the Fick equation and the results evaluated by the Glasgow outcome scale. Measurements of arteriojugular oxygen difference were carried out in 30 patients with subarachnoid haemorrhage due to rupture of intracranial aneurysms, as an attempt to monitor the relationship between changes in AVDO2, clinical picture, and evolution of the patients. The subarachnoid haemorrhage was diagnosed by CT scan in 17 patients and by lumbar punction in 13 and the diagnosis of arterial vasospasm was carried out by clinical evaluation and confirmed by four vessels angiogram in only eight patients. Eighteen patients were admitted with Hunt \& Hess (H\&H) I/II, seven with H\&H III and five with H\&H IV N. Nineteen patients had AVDO2 normal and this group had three deaths; five patients had AVDO2 continuously low with three deaths; and six patients had AVDO2 continuously high with two deaths.

Estudo realizado na Unidade de Neurocirurgia (UNC) e Unidade de Terapia Intensiva (UTD) do Hospital de Base do Distrito Federal (HBDF): *Médico da UNC do HBDF; **Médico da UTI do HBDF; ***Ex-Médico Residente de Neurocirurgia do HBDF; ****Professor Titular de Neurocinurgia da Universidade de Brasilia Aceite: 3-setembro-1996. 
The patients with normal AVDO2 had better prognosis and clinical evolution than the patients with abnormal values of AVDO2. In conclusion, AVDO2 measurements could not be correlated with the diagnosis of vasospasm, but was useful in the early identification of metabolic changes that occur after subarachnoid haemorrhage and could be used as an supplementary monitoring in the clinical evaluation of patients with this pathology.

KEY WORDS: subarachnoid haemorrhage, cerebral aneurysm, arterial vasospasm, cerebral blood flow, arterious venous oxygen difference (AVDO2).

A ruptura de aneurismas intracranianos é a causa mais comum de hemorragia subaracnóidea espontânea (HSAE) e, frequentemente, tem efeitos devastadores para o paciente..$^{23}$ Uma combinação de fatores pode contribuir para os distúrbios na função cerebral seguindo a HSA $5^{8,10,21}$ : direta exposição do tecido cerebral ao sangue extravasado do leito arterial, alterando assim o metabolismo cerebral normal; aumento da pressão intracraniana e distorção mecânica das estruturas intracranianas resultante da injeção rápida de sangue dentro do espaço subaracnóideo; isquemia do tecido cerebral provocado pelo espasmo dos vasos cerebrais. Em consequência dessas alterações ocorre diminuição do aporte de glicose e oxigênio oferecido normalmente pela circulação sanguínea na área cerebral comprometida. As exigências energéticas necessárias para ativar as trocas iônicas, a síntese, a estocagem e a liberação de neurotransmissores, bem como para a manutenção da estrutura tecidual, são fornecidas quase inteiramente pelo metabolismo da glicose a $\mathrm{CO} 2 \mathrm{e} \mathrm{H}_{2} \mathrm{O}^{7,8.24}$. Diferente do músculo e outros tecidos, o cérebro tem somente uma pequena reserva de glicose, glicogênio e ATP, necessitando de um bom fluxo sanguíneo para satisfazer suas necessidades imediatas de energia ${ }^{4,24}$. $O$ comprometimento do fluxo sanguíneo cerebral (FSC) ocasiona rápido consumo das reservas energéticas que são suficientes para manter as necessidades metabólicas por somente alguns minutos. A correção do distúrbio deve ser imediata, antes que a lesão cerebral se torne definitiva ${ }^{2-7}$.

O monitoramento de sinais clínicos em pacientes inconscientes oferece somente informações tardias da deterioração cerebral. ${ }^{9}$ Um método útil para detectar sofrimento cerebral deveria ser sensível o suficiente para demonstrar alteraçōes antes da lesāo neurológica ser reconhecida. ${ }^{27}$ Desde que a maioria da energia requerida pelo cérebro é derivada da glicólise oxidativa e os estoques de oxigênio cerebral sāo insignificantes, devendo ser rapidamente equilibrados pelo afluxo de sangue,, 24 as alterações no consumo cerebral de oxigênio podem ser úteis para detectar comprometimento no metabolismo cerebral, antes da instalação de lesōes neurológicas definitivas ${ }^{4,10,22,25}$. Neste sentido, a medida da diferença artério-venosa de oxigênio (DAVO2) tem sido utilizada como um índice do metabolismo cerebral que se altera precocemente em resposta a uma lesão cerebral. Esta medida consiste na determinação da diferença dos conteúdos de oxigênio em amostras de sangue colhidas simultaneamente na veia jugular comum direita e em uma artéria periférica ${ }^{22.25}$. Dá-se preferência à veia jugular comum direita devido a receber esta a maioria da drenagem venosa do seio sagital superior ${ }^{18}$ e ter representatividade quanto à extração real de oxigênio pelo tecido cerebral, já que a contaminação pela drenagem venosa extracraniana representa 3 a $7 \%$ do total do sangue da veia jugular. ${ }^{19}$ Além disso, a técnica de punção dos vasos, após alguma prática, não é difícil e a complicação mais comum é o hematoma cervical que näo chega a comprometer o estado geral do paciente. Sendo um método simples e pouco dispendioso, pode ser realizado a nível de enfermaria. ${ }^{7}$

Tem sido sugerido que uma diminuição da saturação de oxigênio no sangue colhido na veia jugular que cause grande diferença na DAVO2 é indicativo de isquemia cerebral. ${ }^{9}$ A medida da DAVO2 pode também ser usada como método indireto de avaliação das modificaçōes do FSC em determinado momento da evolução clínica do paciente ${ }^{1213,26}$. Há relação inversa, não linear, entre FSC e DAVO2. Quando o FSC diminui, a DAVO2 aumenta, sugerindo aumento da quantidade de O2 extrá́do do sangue, devido ao reduzido FSC. Este mecanismo protegeria o tecido neuronal, até o FSC ser reduzido, ao ponto da isquemia ou infarto se instalar. Neste ponto, a produção de lactato no cérebro pode ser elevada e demonstrada por um aumento de ácido láctico na amostra venosa ${ }^{22,25}$. Por outro lado, tem sido demonstrado que, em casos de vasoespasmo severo, pode ocorrer aumento da DAVO2 paralelamente à queda do FSC, podendo também traduzir um aumento da resistência 
vascular cerebral (RVC). ${ }^{13}$ Uma redução da DAVO2 pode ser sinônimo de perfusão luxuriante (que pode representar também um sinal de isquemia prévia). A avaliação da DAVO2 tem sido também utilizada como um parâmetro importante no acompanhamento de pacientes com traumatismo crânioencefalico ${ }^{1-7} \mathrm{e}$ em pacientes comatosos de várias causas. ${ }^{26}$

O propósito deste estudo foi avaliar a DAVO2 como um parâmetro útil na conduta clínica e no prognóstico de pacientes com HSAE por ruptura de aneurisma intracraniano.

\section{MATERIAIS E MÉTODOS}

Durante o periodo de janeiro a abril de 1994 foram acompanhados 30 pacientes consecutivos com HSAE, admitidos com ate 72 horas do ictus, independentemente da condiçāo clínica que variou de I a IV na escala de Hunt \& Hess (H\&H). " Dezoito pacientes eram do sexo feminino e 12 do sexo masculino. De acordo com a escala de H\&H, 18 encontravam-se à admissão no grupo I ou II, 7 no grupo III e 5 no grupo IV ou V. A confirmação diagnóstica da HSAE foi realizada pela tomografia de crânio (CT) em 17 pacientes e pela punção lombar em 13 pacientes. Posteriormente, em média após o décimo dia, os pacientes foram submetidos ao estudo angiográfico dos quatro vasos, através da técnica de punção da arteria femural, para elucidação da causa da hemorragia. A presença de aneurisma foi confirmada pela arteriografia em 26 pacientes e através da necrópsia em 4 . As localizações dos aneurismas foram nas artérias carótida interna (13 pacientes), comunicante anterior ( 9 pacientes), na artéria cerebral média ( 7 pacientes) e na fossa posterior (1 paciente). Durante o periodo de intemação, os pacientes receberam tratamento usual para HSAE: foram intubados e ventilados mecanicamente quando necessário, receberam monitorizaçāo contínua de ECG e pressåo arterial sistêmica. Quando submetidos a cirurgia, esta ocorreu, em geral, após a segunda semana do episódio de HSAE.

A coleta do sangue venoso foi realizada através da punção percutânea da veia jugular interna direita, cerca de $2 \mathrm{~cm}$ abaixo da ponta da mastóide, lateralmente à borda medial do músculo estemocleidomastóideo. A coleta do sangue arterial foi feita, preferencialmente, através da punção percutânea da artéria radial. As amostras foram colhidas simultaneamente e submetidas à análise gasométrica convencional, utilizando-se aparelho ABL 330 - Radiometer - Copenhagen; a medida da hemoglobina foi feita pelo aparelho Coulter T-890 - Rio de Janeiro.

A medida da diferença artério-venosa de oxigênio consistiu no cálculo diferencial da extraçăo de oxigênio pelo tecido cerebral através da gasometria do sangue colhido na veia jugular interna e em uma artéria periférica, baseados no princípio de Fick ${ }^{1726}$, através das formulas:

$$
\begin{aligned}
& \mathrm{DAVO} 2=\mathrm{CaO} 2-\mathrm{CjO} 2 \\
& \mathrm{CaO} 2=\mathrm{Hg} \times \mathrm{K}(1,39) \times \mathrm{SAT} \mathrm{O} 2 / 100+\mathrm{PaO} 2 \times \mathrm{K} \\
& \mathrm{CjO} 2=\mathrm{Hg} \times \mathrm{K}(1,39) \times \mathrm{SAT} 2 / 100+\mathrm{PjO} 2 \times \mathrm{K}
\end{aligned}
$$

sendo: $\mathrm{CaO} 2$, conteúdo de oxigênio arterial; $\mathrm{CjO} 2$, conteúdo de oxigênio do sangue obtido na veia jugular; $\mathrm{Hg}$, hemoglobina; SAT O2, saturaçāo de oxigênio da oxi-hemoglobina; $\mathrm{PaO} 2$, pressăo parcial de oxigênio arterial; PjO2, pressão parcial de oxigênio do sangue obtido na veia jugular; $K$, constante 0,0031 .

Os valores da DAVO2 foram considerados normais quando se situavam entre 4,9 a $7 \mathrm{ml} / \mathrm{dl}$. Os valores abaixo de 4,9 ml/dl foram considerados como padrão de hiperemia (perfusão luxuriante) e aqueles acima de 7 $\mathrm{ml} / \mathrm{dl}$ foram considerados como padrāo de hipoperfusāo. ${ }^{2}$ De acordo com os valores da DAVO2 os pacientes foram divididos em 5 grupos conforme proposto por Jakobsen e col.: ${ }^{13}$ GRUPO 1, DAVO2 sempre normal; GRUPO 2, DAVO2 inicialmente baixa, normalizando-se em seguida; GRUPO 3, DAVO2 inicialmente alta, normalizando-se em seguida; GRUPO 4, DAVO2 sempre baixa; GRUPO 5, DAVO2 tendendo sempre a elevação.

Na avaliaçāo clínica dos pacientes foi utilizada a escala de coma de Glasgow ${ }^{29}$ e na análise dos resultados foi utilizada a escala de sequelas de Glasgow (ESG). ${ }^{16} \mathrm{~A}$ análise estatística foi feita pelo teste do qui-quadrado, sendo considerado significativo um alfa de $5 \%$.

\section{RESULTADOS}

Na Tabela 1 apresentamos a relação entre os resultados obtidos da DAVO2 e a avaliaçăo dos pacientes através da ESG, ${ }^{16}$ classificados em cinco graus: boa recuperação $=5$, déficit leve $=4$, déficit moderado $=3$, déficit severo $=2$ e morte $=1$. Dos 19 pacientes que se situavam nos grupos 1,2 e 3, isto é, que tiveram DAVO2 sempre normal ou que normalizaram este parâmetro durante a internação, cerca de 13 deles $(68,4 \%)$ evoluiram 
Tabela l. Distribuição por grupos de pacientes de acordo com a escala de sequelas de Glasgow (ESG) e a evolução das avalią̧ões da DAVO2.

\begin{tabular}{|c|c|c|c|c|c|c|}
\hline Grupo & $\begin{array}{c}\text { Número } \\
\text { de Pacientes }\end{array}$ & ESG 5 & ESG 4 & ESG 3 & ESG 2 & ESG 1 \\
\hline 1 & 12 & 8 & 1 & - & - & 3 \\
\hline 2 & 4 & 3 & 1 & - & - & - \\
\hline 3 & 3 & 2 & 1 & & - & - \\
\hline 4 & 5 & 1 & 1 & - & - & 3 \\
\hline 5 & 6 & 1 & 3 & & - & 2 \\
\hline Total & 30 & 15 & 7 & - & - & 8 \\
\hline
\end{tabular}

Grupos: 1, DAVO2 sempre normal; 2 , inicialmente baixa, normalizando-se em seguida; 3, inicialmente alta, normalizando-se em seguida; 4 , sempre baixa; 5 , sempre alta.

Tabela 2. Mortalidade nos diferentes grupos de acordo com alterações na medida da DAVO2, como definido em Material e Métodos e Tabela $\mathrm{l}$.

\begin{tabular}{ccc}
\hline Grupos & Número de pacientes & Óbitos \\
\hline $1 / 2 / 3$ & $19(63,3 \%)$ & $3(15,8 \%)$ \\
$4 / 5$ & $11(36,7 \%)$ & $5(45,0 \%)^{*}$ \\
Total & $30(100,0 \%)$ & $8(26,6 \%)$ \\
\hline
\end{tabular}

tp<0,05 em relação grupos $1 / 2 / 3$.

Tabela 3 . Ausência de relaçāo entre vasoespasmo cerebral demonstrado pela angiografia e alterações significativas nos valores da DAVO2.

\begin{tabular}{lccc}
\hline & & \multicolumn{2}{c}{ DAVO2 (ml/d) } \\
Vasoespamo & Número de pacientes & $1^{\text {* }}$ coleta (média) & $2^{\mathbf{2}}$ coleta (média) \\
\hline Severo & 3 & 5,3 & 7,0 \\
Moderado & 5 & 5,4 & 6,5 \\
\hline
\end{tabular}

para pontuaçăo de 5 na ESG. Dos restantes seis pacientes, três $(15,7 \%)$ obtiveram pontuações 3 ou 4 e três evoluíram para pontuaçðes 2 ou 1 . A diferença é estatisticamente significativa entre os primeiros grupos $(1,2,3)$ e os restantes ( $\mathrm{p}<0,05$ ). Dos 11 pacientes que tiveram DAVO2 sempre baixa (grupo 4) ou sempre alta (grupo 5), somente dois $(18,1 \%)$ evoluíram para pontuação de 5 na ESG, quatro $(36,3 \%)$ para pontuaçōes 4 ou 3 e os cinco pacientes restantes evoluíram para pontuação de 2 ou $1(45,6 \%)$.

Como apresentado na Tabela 2, também a mortalidade foi estatisticamente maior nos grupos 4 e $5 \mathrm{em}$ relação aos três outros grupos em conjunto $(\mathrm{p}<0,05)$. A análise da relaçāo entre condição clínica, através da 
escala de Hunt \& Hess, ${ }^{11} \mathrm{e}$ os valores de DAVO2 demonstra que os pacientes que se encontravam nos graus I e II mantiveram valores normais da DAVO2. Estes pacientes apresentaram evoluçāo clínica melhor quando comparados com os pacientes que apresentaram modificações desses valores.

O vasoespasmo, confirmado pelo exame radiológico, foi observado em 8 pacientes (Tabela 3). No entanto, não houve relação direta entre a presença de vasoespasmo nestes pacientes e alteraçôes nos valores da DAVO2, já que esta análise esteve sempre dentro da normalidade.

Naqueles pacientes que apresentaram piora do estado neurológico, esta foi precedida por alteração da DAVO2, assim como foram observadas normalizaçōes dos valores da DAVO2 naqueles que evoluíram com melhora clínica. Os seguintes pacientes ilustram esses dados.

Caso 1. MAS, 47 anos, sexo feminino, foi admitida na Unidade de Neurocirurgia do HBDF após dois dias do quadro ictal. Apresentava-se consciente, orientada, com cefaléia intensa e sinais de irritação meníngea, sem sinais focais. A punção lombar mostrou líquor hemorrágico. A DAVO2 feita na ocasião mostrou padrão de hipoperfusão $(7,8 \mathrm{ml} / \mathrm{dl})$. A paciente evoluiu com piora importante do nível da consciência e nova DAVO2 mostrou piora desta relação $(9,4 \mathrm{ml} / \mathrm{dl})$. Iniciada a terapia dos $3 \mathrm{H}$ (hipervolemia, hemodiluição e hipertensão arterial), ${ }^{25}$ ocorreu reversão do quadro clínico e melhora do nível de consciência. Em seguida à instituição da terapêutica e associada à melhora clínica da paciente, observou-se progressiva melhora do padrão da DAVO2 que chegou a normalizar-se $(5,2 \mathrm{ml} / \mathrm{dl})$. No entanto, dois dias após houve deterioraçāo súbita do quadro neurológico que culminou com o óbito. A autópsia demonstrou novo sangramento por ruptura de aneurisma da artéria comunicante posterior.

Caso 2. ALMR, 42 anos, sexo feminino, foi admitida no segundo dia após o ictus, apresentando-se consciente, orientada e referindo cefaléia intensa. Havia sinais de irritação meningo-radicular, mas sem sinais de comprometimento de longas vias. Submetida a CT de crânio, demonstrou-se a presença de sinais compatíveis com HSAE e à angiografia encontrou-se um aneurisma da ACI. No mesmo dia da admissão foi realizada a cirurgia com clipagem do aneurisma. A DAVO2 realizada na admissāo mostrou padrāo de hiperemia (1,8 $\mathrm{ml} / \mathrm{dl}$ ). No primeiro dia pós-operatório, o valor da DAVO2 foi $2,6 \mathrm{ml} / \mathrm{dl}$, mas a paciente apresentava-se no grau II de H\&H. No segundo dia pós-operatório houve melhora clínica (H\&H I) que foi acompanhada da melhora da DAVO2 que se manteve, a partir dá, dentro dos padrōes normais. A paciente teve boa evolução, com alta sem sequelas.

Caso 3. JBT, 50 anos, sexo masculino, com história de cefaléia súbita sendo admitido bastante sonolento, com sinais de irritação meningo-radicular intensos e sem sinais de comprometimento das longas vias (H\&H III). A CT de crânio mostrou hemorragia subaracnóidea abundante. A DAVO2 feita no segundo dia do ictus mostrava padrão de perfusāo luxuriante $(2,3 \mathrm{ml} / \mathrm{dl})$. No dia seguinte houve piora do valor da DAVO2 $(8,2 \mathrm{ml} / \mathrm{dl})$ associada a piora clínica (H\&H IV), culminando com o óbito do paciente no quinto dia após a internação.

\section{DISCUSSÃO}

Os dados apresentados no presente estudo sugerem que a medida seriada da DAVO2, em portadores de HSAE devido à ruptura de aneurisma cerebral, pode representar um parâmetro importante na avaliação do prognóstico desses pacientes. Conforme apresentado na Tabela 1, os 19 pacientes que tiveram DAVO2 sempre normal ou que a normalizaram durante a internação, apresentaram significativamente melhores resultados na escala de sequelas de Glasgow. Os três pacientes que tiveram pontuações de 2 ou 1 foram vítimas de ressangramento e morte súbita. Em contraste, os 11 pacientes situados nos grupos 4 (DAVO2 sempre baixa) e 5 (DAVO2 sempre alta), evoluíram com piores resultados de acordo com a mesma escala. Estas observaçōes estāo de acordo com as de vários autores, confirmando que a DAVO2 é um bom índice para avaliar pacientes com lesão cerebral devido a HSAE ${ }^{10.14,26}$.

A grande demanda de energia requerida pelo cérebro e a obrigatoriedade desta energia ser derivada do metabolismo oxidativo da glicose fazem com que o tecido cerebral seja completamente dependente da continuidade do suprimento sanguíneo. Isto porque as reservas cerebrais tanto de glicose quanto de oxigênio são insignificantes. ${ }^{24}$ Qualquer alteraçāo no fluxo destes elementos, através da corrente sanguínea, pode comprometer a homeostase cerebral ${ }^{8,12-15,17,25,26}$. O FSC é controlado, em 
circunstâncias fisiológicas, pela RVC. Esta última depende da taxa metabólica de consumo de oxigênio, da pressão arterial de oxigênio, da pressão arterial de gás carbônico e da pressão de perfusão cerebral. Comprometimento em qualquer um destes parâmetros, dependendo da intensidade e da associação entre eles, ocasiona alteraçōes no FSC (discussão e referências ${ }^{2-7}$ ). Por outro lado, se existe lesão importante do tecido cerebral este pode tornar-se incapaz de realizar o metabolismo energético normal. ${ }^{2} \mathrm{Em}$ qualquer dessas condiçōes podemos ter diminuição do consumo de oxigênio pelo cérebro e, em consequência, alteração da DAVO2.

Durante a evolução da HSAE, o FSC se altera com o tempo em função do aumento da pressāo intracraniana, da hiperemia transitória e do vasoespasmo das artérias cerebrais ${ }^{10,21}$. Após uma HSAE, o FSC está diminuido e pode piorar à medida que o quadro clínico evolui. A causa para esta modificação global no FSC é desconhecida e não parece estar relacionada ao vasoespasmo angiográfico. ${ }^{10}$ No entanto, diversos autores também observaram redução paralela na taxa metabólica de consumo de oxigênio e FSC, com a piora clínica do vasoespasmo. A opinião comum, entretanto, é que a depressão global no fluxo não pode ser atribuída diretamente ao vasoespasmo. Em vez disto, esta redução no FSC pode estar relacionada à taxa metabólica de consumo de oxigênio deprimida. A redução desta taxa é a maior consequencia da HSAE. Dependendo da intensidade destes fatores o quadro clínico do paciente pode deteriorar-se e sua evolução ficar comprometida. Todas estas causas podem contribuir para alterações no metabolismo cerebral expressado nas alterações da DAVO2. Por exemplo, em um modelo de hipertensão intracraniana em gatos, a DAVO2 e o metabolismo energético cerebral, medido pela espectroscopia com fósforo 31 , correlacionou-se bem com vários niveis de hipertensão intracraniana ${ }^{28} \mathrm{Em}$ várias casuísticas tem sido demonstrado que a clevação da pressão intracraniana pode cursar com aumento da DAVO2,4,14,20. Em alguns casos ocorre normalizaçāo da DAVO2 após drenagem ventricular. ${ }^{13} \mathrm{Tem}$ sido também descrito uma correlaçāo positiva entre quantidade de sangue demonstrada pela $\mathrm{CT}$ e a reduçāo na captaçāo de oxigênio pelo cérebro. ${ }^{15} \mathrm{Tal}$ correlação ocorreu no Caso 3 do presente estudo em que, associadamente a intensa hemorragia subaracnóidea, havia alterações persistentes na DAVO2.

A presença de sangue fora dos vasos cerebrais, ocasionando uma série de mudanças no metabolismo cerebral, pode ser responsável pela inabilidade dos neurônios $\mathrm{em}$ usar o oxigênio que é fornecido, devido a disfunção mitocondrial ou distúrbios na atividade enzimática ${ }^{2,8}$. Quanto maior a alteração na dificuldade das células cerebrais $\mathrm{cm}$ metabolizar a glicose, através das vias oxidativas, maior é a possibilidade de uma lesāo definitiva seguindo a HSAE. Este fato é demonstrado pelos presentes dados em que vemos uma correlação positiva entre DAVO2 persistentemente alterada (Tabela 1) e pobre escore na escala de sequelas de Glasgow. Tamberm a DAVO2 sempre alterada correlaciona-se com a maior mortalidade encontrada nestes grupos de pacientes em relação aos grupos restantes com a DAVO2 normal ou que a normalizaram. A cirurgia precoce, realizada no Caso 2, em que houve drenagem do sangue ventricular, ocasionou melhora acentuada do quadro clínico do paciente, associada a melhora progressiva da DAVO2.

Em nossa casuística, somente oito pacientes tiveram vasoespasmo cerebral seguindo a HSAE, devidamente comprovado pela angiografia cerebral, único exame disponivel em nosso hospital para documentar esta complicação. No entanto, nenhum deles apresentou alterações na DAVO2 (Tabela 3). Este dado não é surpreendente, já que um vasoespasmo severo, para ocasionar alterações hemodinâmicas importantes, deve comprometer mais de $70 \%$ do diâmetro do vaso ${ }^{12} \mathrm{e}$ vasoespasmo cerebral sintomático ocorre em apenas 20 a $30 \%$ dos pacientes com HSAE. ${ }^{25}$ Além do mais, vasoespasmo suficiente para reduzir, de maneira significativa, o FSC ocorre em menos de $10 \%$

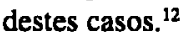

De fato, vários estudos têm mostrado baixa incidência da associação entre vasoespasmo cerebral e DAVO2 anormalmente elevada. Jakobsen e col. ${ }^{14}$ encontraram $7,1 \%$ desta associaçăo em portadores de HSAE. Outros observaram somente em 5 de 45 pacientes. ${ }^{13} \mathrm{Já}$ Voldby e col. ${ }^{20,30}$ não mostraram nenhuma alteração da DAVO2 em 31 pacientes com vasoespasmo cerebral. No entanto, o Caso 3 do presente estudo, apesar de não ter sido estudado radiologicamente para demonstrar o clinicamente 
presente vasoespasmo cerebral, respondeu à terapia específica com melhora clínica e normalização da DAVO2. Este caso sugere que um vasoespasmo importante pode alterar a DAVO2. Nesta situação, o vasoespasmo aumentaria a RVC, levando a diminuição do fluxo sanguíneo e do conteúdo cerebral de oxigênio ${ }^{14,31}$.

Uma possível explicação para esta pouco frequente associação entre alteração na DAVO2 e vasoespasmo seria o desencadeamento de um mecanismo compensatório ao vasoespasmo. A ocorrência deste em grandes vasos provocaria dilatação das pequenas arteríolas intracerebrais, com a consequente diminuição da RVC periférica e aumento do FSC. Assim, o suporte sanguíneo seria mantido e garantiria a oferta de oxigênio e glicose, contribuindo para a DAVO2 quase sempre normal nestes pacientes (discussão mais detalhada e referências, em Jakobsen e col. ${ }^{13}$ ).

Por outro lado, a medida da DAVO2 isolada, como a realizada no presente estudo, apresenta às vezes restrições na sua interpretação. Por exemplo, tem sido demonstrado que em pacientes em coma agudo traumático, associado a anemia aguda, a DAVO2 pode estar falsamente diminuida, sugerindo hiperemia cerebral ${ }^{2.35}$. Tendo esta limitação em vista, muitos autores preconizam a adoção de extração cerebral de oxigenio, definida como a diferença entre as saturações arterial e do bulbo jugular de oxi-hemoglobina, por ser mais fidedigna para a monitorização fisiologica em pacientes com ou sem anemia aguda ${ }^{2,3.5}$. Outros parâmetros para a monitorização da oxigenação cerebral têm sido preconizados (consultar a excelente revisão de $\mathrm{Cruz}^{2}$ ). No entanto, medidas de FSC ou monitorização contínua com catéter intrajugular, apesar de serem mais fidedignas, apresentam ainda limitações técnicas em nosso meio.

Em conclusão, os dados aqui apresentados sugerem que alteraçōes na DAVO2 correlacionamse bem com o quadro clínico presente no momento do exame e que a pesquisa sistemática deste parâmetro pode fornecer uma indicaçāo sobre o prognóstico desses pacientes, além de auxiliar no monitoramento clínico a nivel de enfermaria, facilitando com isto a tomada de decisōes quanto ao momento ideal da realizaçāo de intervençōes cirúrgicas e exames invasivos.

\section{REFERÊNCIAS}

1. Bouma GJ, Muizelaar JP, Choi SC, Newlon PG, Young HF. Cerebral circulation and metabolism after severe traumatic brain injury: the elusive role of ischemia. J Neurosurg 1991;75:685-693.

2. Cruz J. Monitorizaçāo hemodinâmica e metabólica cerebral em humanos. Arq Bras Neurocirurg 1993:11:209-215.

3. Cruz J. Combined continuous monitoring of systemic and cerebral oxygenation in acute brain injury: preliminary observations. Crit Care Med 1993;21:1225-1232.

4. Cruz J. An additional therapeutic effect of adequate hyperventilation in severe acute brain trauma: normalization of cerebral glucose uptake. J Neurosurg 1995;82:379-385.

5. Cruz J, Jaggi JL, Hoffstad OJ. Cerebral blood flow and oxygen consumption in acute brain injury with acute anemia: an alternative for the cerebral metabolic rate of oxygen consumption? Crit Care Med 1993;21:1218-1224.

6. Cruz J, Jaggi JL, Hoffstad OJ. Cerebral blood flow, vascular resistance, and oxygen metabolism in acute brain trauma: redefining of cerebral perfusion pressure? Crit Care Med 1995;23:1412-1417.

7. Cruz J, Raps EC, Hoffstad OJ, Jaggi JL, Gennarelli TA. Cerebral oxygenation monitoring. Crit Care Med 1993;21:1242. 1243.

8. Fein JM. Cerebral energy metabolism after subarachnoid haemorrhage. Stroke 1975;6:1-8.

9. Garlick R, Bihari D. The use of intermittent and continuous recordings of jugular venous bulb oxygen saturation in the unconscious patient. Scand J Clin Lab Invest 1987;47(Suppl 188):47-52.

10. Grubb RL, Raichle ME, Eichling JO, Gado MH. Effects of subarachnoid haemorrhage on cerebral blood volume, blood flow, and oxygen utilization in humans. J Neurosurg 1977;46:446-453.

11. Hunt WE, Hess RM. Surgical risk as related to time of intervention in the repair of intracranial aneurysms. J Neurosurg 1968;28:14-20.

12. Jakobsen $M$, Enevoldsen EM, Bjerre P. Cerebral blood flow and metabolism following subarachnoid haenornage; cerebral oxygen uptake and global blood flow during the acute period in patients with SAH. Acta Neurol Scand 1990; 82:174-182.

13. Jakobsen M, Enevoldsen EM, Dalager T. Spasm index in subarachnoid haemorrhage: consequences of vasospasm upon cerebral blood flow and oxygen extraction. Acta Neurol Scand 1990;82:311-320.

14. Jakobsen M, Overgaard J, Marcussen E, Enevoldsen EM. Relation between angiographic cerebral vasospasm and regional CBF in patients with SAH. Acta Neurol Scand 1990;82:109-115.

15. Jakobsen M, Skjodt T, Enevoldsen EM. Cerebral blood flow and metabolism following subarachnoid haemorrhage: effect of subarachnoid blood. Acta Neurol Scand 1991;83:226-233. 
16. Jennett B, Bond M. Assesment of outcome after severe brain damage: a practical scale. Lancet 1975;1:480-484.

17. Kaminogo M, Ichikura A, Shibata S, Toba T, Yonekura $M$. Effect of acetazolamide on regional cerebral oxygen saturation and regional cerebral blood flow. Stroke 1995;26:2358-2360.

18. Knuckey NW, Fox RA, Surveyor I, Strokes BAR. Early cerebral blood flow and computarized tomography in predicting ischemia after cerebral aneurysm rupture. J Neurosurg 1985;62:850-855.

19. Lassen NA, Lane MH. Validity of internal jugular blood for study of cerebral blood flow and metabolism. J Appl Physiol 1961;16:313-320.

20. Mathew NT, Meyer JS, Hartmann A. Diagnosis and treatment of factors complicating subarachnoid haemorrhage. Neuroradiology 1974;6:237-245.

21. Meyer CHA, Lowe D, Meyer M, Richardson PL, Neil-Dwyer G. Progressive change in cerebral blood flow during the three weeks after subarachnoid haemorrhage. Neurosurgery 1983;12:58-76.

22. Muizelaar JP, Marmarou A, Ward JD, Kontos HA, Choi SC, Becker DP, Gruemer H, Young HF. Adverse effects of prolonged hyperventilation in patients with severe head injury: a randomized clinical trial. J Neurosurgery 1991;75:731-739.

23. Pulsinelli WA, Levy DE. Cerebrovascular disease. In Wyngaarden JB, Smith LH, Bennett JC (eds.). Cecil textbook of medicine. Ed. 19. Philadelphia; Saunders;1992:2145-2162.

24. Ritter AM, Robertson CS. Intensive care management of the neurosurgical patients. Contemp Neurosurg 1994;16:1 -8.

25. Robertson CS, Narayan RK, Gokaslan ZL, Pahwa R, Grossman RG, Caram P, Allen E. Cerebral arteriovenous oxygen difference as an estimate of cerebral blood flow in comatose patients. J Neurosurg 1988;70:222-230.

26. Sheinberg M, Kanter MJ, Robertson CS, Contant CF, Narayan RK, Grosst.an RG. Continuous monitoring of jugular venous oxygen saturation in head-injured patients. J Neurosurg 1992;76:212-217.

27. Sutton LN, McLaughlin AC, Dante S. Cerebral venous oxygen content as a measure of brain energy metabolism with increased intracranial pressure and hyperventilation. J Neurosurg 1990;73:927-932.

28. Teardale G, Jennett B. Assesment of coma and impaired consciousness: a practical scale. Lancet 1974;2:81-84.

29. Voldby B, Enevoldsen EM. Intracranial pressure changes following aneurysm rupture: Part 3. Recurrent haemorrhage. J Neurosurg 1982;56:784-789.

30. Voldby B, Enevoldsen EM, Jensen FT. Regional cerebraJ blood flow, intraventricular pressure and cerebral metabolism in patients with ruptured intracranial aneurysms. J Neurosurg $1985 ; 62: 48-58$. 